\title{
Etiología de la ginecomastia. Importancia de no subdiagnosticar una ginecomastia patológica
}

\author{
Enzo Devoto $C^{1}$, Marcia Madariaga A $^{2 a}$, Lucía Aravena ${ }^{1}$, \\ Ximena Lioi $C^{1}$.
}

\section{Etiological study of gynecomastia. Results of a prospective study and recommendations}

Background: Gynecomastia can be physiological or pathological. A limited study of gynecomastia is recommended during puberty and in the elderly, ages in which gynecomastia is usually considered physiological. Other authors suggest that this condition should be studied when it is painful, rapidly growing, of recent onset, when its diameter is more than $4 \mathrm{~cm}$ and when is associated to testicular masses. Aim: To investigate the causes of gynecomastia and to evaluate the above mentioned criteria to exclude pathological conditions. Material and methods: Prospective study of 117 patients aged 10 to 83 years, consulting for gynecomastia. All were subjected to a standardized study including a clinical examination and measurement of plasma estradiol and testosterone levels. Results: Forty one percent of gynecomastias were considered pathological and the rest, physiological. Among pathological conditions, 18 patients had an endocrine etiology (hypogonadism in ten patients, estrogen secreting tumors in three, hyperestrogenism of unknown etiology in four and peripheral resistance to androgens in one), in 17 it was secondary to medications and in 13 it was secondary to other causes (idiopathic, pesticide exposure, alcoholism, diabetes or re feeding). In $79 \%$ of 86 patients of less than 20 years, the condition was physiological and in four of five elderly subjects, it was pathological. Thirty nine percent of pathological gynecomastias lacked the signs and symptoms that according to authors, should prompt a thorough study. Conclusions: All patients with gynecomastia should be studied with a complete medical history and the measurement of estradiol and testosterone levels. The criteria proposed to conduct minimal studies in gynecomastia, would miss a large volume of pathological conditions (Rev Méd Chile 2007; 135: 189-97).

(Key words: Estrogens; Gynecomastia; Hypogonadism)

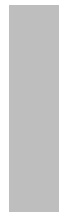

Recibido el 20 de julio, 2005. Aceptado el 3 de agosto, 2006.

${ }^{1}$ Sección Endocrinología, Servicio de Medicina, Hospital Clínico San Borja Arriarán, Departamento de Medicina, Facultad de Medicina, Campus Centro, Universidad de Chile. ${ }^{2}$ Instituto de Investigaciones Materno Infantil, Facultad de Medicina, Campus Centro, Universidad de Chile . Santiago de Chile

aTecnólogo Médico

Correspondencia a: Dr. Enzo Devoto C. Hernando de Aguirre 194 Of. 62. Fax: 2290238. Santiago, Chile. E mail: edevoto@vtr.net 
$\mathrm{L}^{\mathrm{a}}$ a ginecomastia (Gi), aumento de tamaño de la mama en el hombre, es un signo clínico y no una enfermedad ${ }^{1,2}$. Se produciría por desbalance plasmático o intramamario de estrógeno-testosterona (E-T): los estrógenos aumentan por mayor secreción funcional, tumoral o estrógenos exógenos y los andrógenos disminuyen por una alteración que afecta cualquier nivel del eje hipotálamo-hipófisis-testículo (HHT) ${ }^{3}$.

Se produciría un desbalance E-T intramamario, por: 1. Síntesis estrogénica in situ, debida a mayor expresión de las enzimas aromatasas, que transforman andrógenos en estrógenos. 2. Menor acción androgénica local, debida a alteraciones del receptor de andrógenos o antagonismo de antiandrógenos ${ }^{4}$.

La Gi es un problema frecuente en púberes y adolescentes, con prevalencia de 19\% a $70 \%$ y mayor frecuencia entre los 14 y 15 años. En 90\% de los casos desaparece espontáneamente en un período de 3 años ${ }^{5-7}$. En el adulto sano, se estimaba poco frecuente, aunque estudios en personal militar, hospitalizados, tercera edad y autopsias, revelan prevalencia entre $35 \%$ y $65 \%^{8-10}$.

Estas diferencias en prevalencia se explicarían por los criterios de diagnóstico utilizados, unos consideran $\mathrm{Gi}$ sobre $0,5 \mathrm{~cm}^{3,5}$ y otros sobre 2,0 $\mathrm{cm}^{3,5,8,10}$.

La Gi fisiológica es prevalente en los períodos neonatal, pubertad y adulto mayor, debe diferenciarse de la patológica, cuya causa debe ser diagnosticada y tratada (Tabla 1).

Hay discusión entre los autores respecto a cómo estudiar una Gi. Algunos solicitan inmediatamente exámenes de laboratorio, hormonas, marcadores tumorales e imágenes, lo que sería de alto costo y bajo rendimiento, por el reducido porcentaje de tumores funcionantes causantes de $\mathrm{Gi}^{11}$.

Otros recomiendan realizar estudio ante portadores de Gi que presenten síntomas y signos

Tabla 1. C ausas de ginecomastia

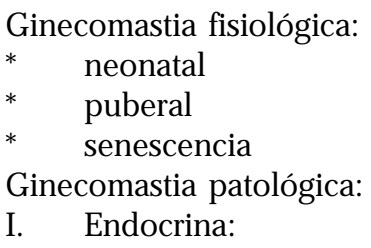

I. Endocrina:

A) Hiperestrogenismo

* Tumores secretores de estrógenos (leydigioma feminizante, tumor de células de Sertoli)

* Tumores secretores de HCG (seminoma, coriocarcinoma y otros)

* Tumores adrenales feminizantes

* Aumento de producción de estrógenos por incremento de actividad de aromatasas (por causa genética o secundaria a otros estados patológicos)

B) Disminución de síntesis de testosterona

* Falla gonadal primaria: congénita (Klinefelter) adquirida (infecciones, orquitis etc)

* Falla testicular por enfermedad hipotalámica o hipofisiaria.

C) Resistencia periférica a andrógenos.

II. Farmacológica

III. Otras:

* Enfermedades renales crónicas

* Enfermedades hepáticas

* HIV

* Realimentación

* Hipertiroidismo

* Alcohol

* Tóxicos 
como dolor, crecimiento rápido, inicio reciente, diámetro mayor de $4 \mathrm{~cm} \mathrm{o}$ masa testicular palpable $1,12-14$.

También se ha planteado que en edades donde la Gi fisiológica es frecuente (pubertad, adolescencia y senescencia) realizar sólo estudio clínico, tranquilizar al paciente y controlarlo posteriormente ${ }^{3,15}$.

Existen estudios nacionales de $\mathrm{Gi}$ en servicios de pediatría y cirugía ${ }^{16,17}$. Como un aporte desde el punto de vista del internista, realizamos un trabajo retrospectivo de una cohorte de pacientes con Gi estudiados en nuestro policlínico con el diagrama de flujo que anexamos al trabajo.

Sus objetivos son investigar las causas de Gi y evaluar la omisión de Gi patológicas, que habría ocurrido si la investigación etiológica se hubiera realizado sólo ante síntomas y signos predeterminados o con investigación somera en grupos etarios donde la Gi puede ser fisiológica.

\section{PACIENTES Y MÉTODO}

Ciento diecisiete pacientes, con rango de edad 10-83 años, consultaron en el Policlínico de Andrología, en el período 1977-2000, referidos por ginecomastia. Todos los pacientes completaron un estudio previamente diseñado, que comprendía anamnesis, examen físico general, segmentario y genital.

En el examen mamario, la presencia de tejido mamario se diferenció del aumento de volumen por tejido adiposo o lipomastia, cogiendo el tejido entre pulgar e índice y comparándolo con igual maniobra realizada a nivel del pliegue axilar anterior. El tejido mamario es firme, muy diferente a la consistencia de la piel y grasa del pliegue axilar $^{10}$. Para determinar el volumen se desplazó el pulgar del examinador (E.D.) desde el pezón hacia la periferia en sentido vertical y transversal; se determinaron los márgenes externo e interno, para el diámetro transversal y superior e inferior, para el vertical. Con regla, se midió la distancia entre el pulgar y el índice ubicados en dichos bordes, estableciéndose en centímetros el diámetro máximo del tejido mamario. Consideramos clínicamente importante una Gi de $2 \mathrm{~cm}$ o más ${ }^{8-10}$.

Se midió el volumen testicular con orquidómetro de Prader y se descartó tumor testicular ${ }^{18}$ por palpación. La pubertad se clasificó de acuerdo a las etapas de Tanner.
Se realizó medición de estradiol $\left(\mathrm{E}_{2}\right)$ y testosterona (T) plasmática, determinados por radioinmunoanálisis específico (Programa OMS). Valores normales: estradiol $<50 \mathrm{pg} / \mathrm{ml}$ y testosterona $2-6 \mathrm{ng} / \mathrm{ml}$.

Otros exámenes de laboratorio se solicitaban sólo en base a la clínica 0 ante un valor de $\mathrm{T}$ o $\mathrm{E}_{2}$ alterados. En caso de sospecha de hipogonadismo y T baja, se solicitó hormona foliculoestimulante (FSH), hormona luteoestimulante (LH) y prolactina (PRL); en el hipogonadismo hipogonadotropo, estudio de imágenes hipotálamo-hipofisiaria; en el hipergonadotropo, cariograma, si se sospechó síndrome de Klinefelter.

Ante aumento de estrógenos plasmáticos, con o sin masa palpable abdominal o testicular, se solicitó estudio ecotomográfico y marcadores tumorales (ßHCG y alfa feto proteína) y test de estimulación con gonadotrofina coriónica humana $(\mathrm{HCG})^{19}$.

Las causas de $\mathrm{Gi}$, se clasificaron de acuerdo a la Tabla 1 en: 1) Gi fisiológica, previa exclusión de otra etiología. 2) Gi patológica: endocrina, farmacológica, secundaria a enfermedad general, alcohol, tóxicos ${ }^{2,12,20}$.

Las $\mathrm{Gi}$ del adulto con anamnesis y examen físico normal y sin alteración de valores de $\mathrm{E}_{2} \mathrm{y} \mathrm{T}$, fueron clasificadas como idiopáticas ${ }^{1,21,22}$.

Analizamos esta casuística y determinamos el porcentaje de Gi fisiológicas y patológicas, el porcentaje de las distintas causas de Gi patológicas; el porcentaje de $\mathrm{Gi}$ patológicas que no presentaban los síntomas y signos sugeridos para que una $\mathrm{Gi}$ amerite ser investigada y el porcentaje de causas patológicas en menores de 20 y en mayores de 60 años.

\section{RESULTADOS}

Características clínicas y de laboratorio del grupo estudiado. La mayor frecuencia de Gi se concentró en el grupo etario de 10 a 20 años, disminuyendo después de los 30; 5\% tenía sobre 50 años.

Las etapas de Tanner en los menores de 20 años eran: 14\% (II), 23\% (III), 39\% (IV) y 30\% (V).

Un paciente inició Gi prepuberal (10 años), 83\% de los puberales en los 2 primeros años de la pubertad. No hubo antecedentes familiares de $\mathrm{Gi}$; la ingesta de alcohol y fármacos fueron considerados factores etiológicos previa exclusión de otras causas. 
Encontramos Gi bilateral en 58\%, mastalgia en $54 \%$ y descarga por el pezón en 3\%.

$\mathrm{Al}$ medir diámetro mayor, en $54 \%(\mathrm{n}=63)$ era menor de $5 \mathrm{~cm}$ y en $46 \%(\mathrm{n}=54)$ era mayor de $5 \mathrm{~cm}$.

Se encontró microorquia en 9 pacientes (hipogonadismos primarios y secundarios), nódulo testicular palpable en 1 (seminoma) y zona de mayor consistencia en otro (tumor de células de Sertoli).

Se encontró testosterona disminuida en 7\% (8/ 117) y estradiol elevado en $11 \%(13 / 117)$.

En 2/8 de los pacientes con $\mathrm{T}$ disminuida y en $6 / 13$ con $E_{2}$ elevado, la anamnesis y examen físico eran normales. Las patologías asociadas a estas alteraciones se describen en la Tabla 2.

Los pacientes con hipogonadismo hipogonadotropo presentaron $\mathrm{LH}$ bajos o $\mathrm{LH}$ inadecuadamente normal para una testosterona baja. FSH y $\mathrm{LH}$ estuvieron elevados en pacientes con falla testicular primaria. No hubo casos de hiperprolactinemia.

En 2 pacientes con tumor testicular, uno de ellos no palpable, el test de estimulación con HCG $^{17}$ demostró una hiperrespuesta patológica del estradiol (>140 pg/ml) a las $24 \mathrm{~h}$ post HCG.

No se realizó estudio de laboratorio tiroideo, por no existir clínica de disfunción tiroidea en ningún paciente.

La ecotomografía testicular diagnosticó tumor no palpable en un paciente con hiperestrogenismo.

Etiología. En nuestra casuística, las causas de Gi fueron: fisiológica en 59\% (68 puberales y 1 del adulto mayor) y patológica en $41 \%(\mathrm{n}=48)$. La Tabla 3 detalla las causas de Gi patológicas, en que destacan la causa endocrina $(\mathrm{n}=18)$, ingesta de fármacos ( $\mathrm{n}=17)$, de éstos, 9 por terapia de acné severo con antiandrógenos y otras causas ( $\mathrm{n}=13$ ).
Las causas endocrinas se debieron a hipogonadismos 0 a hiperestrogenismos. De 10 pacientes con hipogonadismo, 2 fueron hipogonadotropos, uno congénito (Kallmann) y el otro adquirido pospuberal por disfunción hipotalámica, ya que la T subió a lo normal al administrar citrato de clomifeno 23

Los 8 pacientes con hipogonadismo hipergonadotropo, 4 eran síndrome de Klinefelter, 1 varón XX (fenotipo masculino, cariograma $46 \mathrm{XX}$, clínica similar al Klinefelter y gran Gi); 1 postorquitis y 2 de causa no precisada. Los 4 pacientes con Klinefelter presentaban microorquia, FSH y LH elevadas; 2/4 T normal baja y 2 hipotestosteronemia.

De los hiperestrogenismos destacan:

1) Causa no precisada: 1 paciente prepuberal $\left(\mathrm{E}_{2}\right.$ $=60 \mathrm{pg} / \mathrm{ml}$ ) y 3 adultos jóvenes, con $E_{2}$ entre 64 y $68 \mathrm{pg} / \mathrm{ml}$, carecían de antecedentes familiares de $\mathrm{Gi}$, otras enfermedades, ingesta de drogas, alcohol, fármacos o contaminación estrogénica por crema, shampoo etc. Los estudios de imágenes descartaron lesiones tumorales testiculares, suprarrenales, hepáticas 0 pulmonares; los marcadores tumorales como la $ß$ HCG y $\alpha$ fetoproteína fueron negativos.

2) Tres pacientes con hiperestrogenismo correspondieron a tumores testiculares:

2.1. Paciente de 15 años $\left(E_{2}=69 \mathrm{pg} / \mathrm{ml}\right)$, el examen testicular detectó zona de mayor induración y sensibilidad a la presión, orquiectomizado, se diagnosticó tumor de células de Sertoli.

2.2. Paciente de 25 años $\left(E_{2}=102 \mathrm{pg} / \mathrm{ml}\right)$, sin masa palpable testicular ni abdominal, hiperrespuesta al test de HCG; la ecografía demostró masa intratesticular compatible

Tabla 2. Patologías asociadas a alteraciones de hormonas gonadales en ginecomastias observadas en 21 pacientes

\begin{tabular}{ll}
$\begin{array}{ll}\text { Pacientes con } \\
\text { hiperestrogenismo }(\mathrm{n}=13)\end{array}$ \\
\hline Síndrome de Klinefelter & $(3)$ \\
Tumor testicular & $(3)$ \\
Alcoholismo & $(2)$ \\
Ingesta de estrógenos naturales & $(1)$ \\
Ginecomastia prepuberal & $(1)$ \\
Adulto con Gi sin causa identificable & (3)
\end{tabular}

Pacientes con

hipotestosteronismo $(\mathrm{n}=8)$

3) Hipogonadísmo hipogonadotropo

3) Hipogonadísmo hipergonadotropo

(2)

(6) 
Tabla 3. Etiología de las $\mathbf{4 8}$ ginecomastias patológicas

\begin{tabular}{|lr|}
\hline & $\mathrm{n}$ (rango edad) \\
\hline Ginecomastia endocrina & $8(14-62)$ \\
Hipogonadismo hipergonadotropo o falla testicular primaria & $2(19-39)$ \\
Hipogonadismo hipogonadotropo o falla testicular 2o & $3(14-36)$ \\
Tumores (1 seminoma, 1 Sertoli y 1 histología desconocida) & $4(10-26)$ \\
Hiperestrogenismo de causa no precisada & $1(28)$ \\
¿Resistencia periférica a andrógenos? & $9(16-33)$ \\
Ginecomastia farmacológica & $4(25-82)$ \\
Ingesta de antiandrógenos & $4(12-40)$ \\
(cimetidina, flutamida, etinil estradiol-ciproterona, espironolactona) & \\
Ingesta de fármacos de acción estrogénica & $1(37)$ \\
(1 digitálico, 2 etinilestradiol y 1 valerianato de estradiol) & $4(15-20)$ \\
Ingesta de otros fármacos (ketoconazol, anabólicos) & $2(19-46)$ \\
Ginecomastia por otras causas & $2(20-25)$ \\
Pesticidas & $4(27-50)$ \\
Alcohol & \\
Diabetes mellitus tipo 1 & \\
Ginecomastia de la realimentación & \\
Ginecomastia idiopática & \\
\hline
\end{tabular}

con tumor. Se refirió a urólogo, no conociéndose el resultado de la intervención.

2.3. Paciente de 33 años $\left(E_{2}=180 \mathrm{pg} / \mathrm{ml}\right)$ con nódulo testicular palpable, ecografía compatible con tumor, $§$ HCG elevada, estudio histológico: seminoma.

3) De los 4 pacientes con síndrome de Klinefelter anteriormente descritos, sólo 3 tenían $\mathrm{E}_{2}$ elevado: $107,59,98 \mathrm{pg} / \mathrm{ml}$.

4) En 2 de 4 pacientes con alcoholismo, los valores de $\mathrm{E}_{2}$ fueron: 70 y $100 \mathrm{pg} / \mathrm{ml}$; en paciente transexual con ingesta de estradiol, $141 \mathrm{pg} / \mathrm{ml}$.

Un paciente con T y LH elevada sugería resistencia periférica a andrógenos ${ }^{2}$.

Dos Gi ocurrieron en período de recuperación de pacientes afectados en su estado general y nutritivo por politraumatismo severo.

Un portador de Gi sin otra causa demostrable, manipulaba pesticidas sin protección y presentaba daño hepático compatible con acción tóxica del pesticida.

Los 2 pacientes con diabetes mellitus tipo 1 desarrollaron la $\mathrm{Gi}$ al compensárseles luego de años de control deficiente.
En 86 pacientes puberales y adolescentes $(<20$ años), las causas de Gi fueron fisiológicas en 79\% ( $n=68)$ y patológicas en $21 \%(n=18)$. Las causas de $\mathrm{Gi}$ patológicas a esa edad y en los mayores de 60 años se describen en la Tabla 4.

En 39\% $(\mathrm{n}=19)$ de las 48 Gi patológicas no se encontró dolor, crecimiento rápido, inicio reciente, diámetro máximo mayor de $4 \mathrm{~cm}$ ni masa testicular palpable.

\section{DisCUSIÓN}

Se investigó etiología de Gi por semiología y determinación de $\mathrm{E}_{2}$ y T. Los hallazgos de microorquia y la semiología de hipoandrogenismo sugirieron el diagnóstico de hipogonadismo; la palpación de nódulos, mayor consistencia y dolor, el de tumor testicular.

La medición de $\mathrm{T}$ confirmó el diagnóstico clínico de hipogonadismo y permitió diagnosticar aquellos sin clínica y volumen testicular normal; $\mathrm{E}_{2}$ elevado permitió diagnosticar cuadros con hiperestrogenismo, incluso en pacientes con clínica normal. 
Tabla 4. Ginecomastias patológicas en menores de 20 años y en mayores de 60 años

\begin{tabular}{|llll|}
\hline Menores de 20 años $(\mathrm{n}=18)$ & & Mayores de 60 años $(\mathrm{n}=4)$ \\
\hline Fármacos & 8 & Fármacos (digital y estrógenos) & 2 \\
Etanol & 3 & & \\
Klinefelter & 2 & Falla testicular primaria post orquitis & 1 \\
Tumor células de Sertoli & 1 & & \\
Hipogonadismo hipogonadotropo & 1 & Falla testicular primaria & \\
Varón 46 xx & 1 & de causa desconocida & \\
Hiperestrogenismo idiopático & 1 & & \\
Diabetes mellitus & 1 & & \\
\end{tabular}

La semiología y valores hormonales sugirieron la realización de otros estudios, como ecografía ante signos testiculares sospechosos de tumor 0 $\mathrm{E}_{2}$ elevado.

Se solicitó prolactina (PRL) en presencia de hipotestosteronemia ${ }^{14,23}$, sin encontrarse hiperprolactinemia. Esto coincide con autores que no encuentran rol importante de la PRL en $\mathrm{Gi}^{2,3,9,14}$ y difiere de otros ${ }^{24,25}$.

Llama la atención la ausencia de causa tiroidea, ya que históricamente se considera al hipertiroidismo causante de $\mathrm{Gi}^{2,3,7,9,12-14,20,26}$.

El rango etario del grupo estudiado explica el mayor porcentaje de $\mathrm{Gi}$ fisiológicas sobre las patológicas, en estas últimas, el factor endocrino fue diagnosticado con la metódica utilizada y el factor farmacológico, con anamnesis y exclusión de otras causas.

La Gi puede ser debida a un tumor testicular funcionante, lo que ocumió en 2,5\% de los casos, baja frecuencia, coincidente con los resultados de $0 \%$ de Carlson ${ }^{9}$, 2\% de Bowers ${ }^{11}$ y 3\% de Braunstein ${ }^{3}$.

El hiperestrogenismo en el seminoma es debido a la producción de HCG que aumenta la secreción de $\mathrm{E}_{2}$ testicular ${ }^{27}$; en el tumor de células de Leydig feminizante y en el de Sertoli, está incrementada la secreción de estrógenos, habiéndose demostrado en este último mayor expresión de la citocromo P450 aromatasa ${ }^{28}$.

El tumor testicular sin confirmación histológica, diagnosticado por ultrasonografía en etapa no palpable, era compatible con tumor de células de Leydig, ya que la $\mathrm{Gi}$ puede preceder a su palpación y presentar hiperrespuesta a HCG $^{19,29}$.

La Gi prepuberal puede deberse a tumores secretores de estrógenos, HCG o incremento de la aromatización de andrógenos a estrógenos por causa genética ${ }^{11,20,22,30,31}$. En el paciente prepuberal y en los 3 con hiperestrogenismo idiopático del adulto, podría existir aumento de acción de aromatasas, posibilidad que no pudo ser estudiada.

El hipogonadismo hipergonadotropo ${ }^{14,32}$ produce Gi por hipotestosteronismo o hiperestrogenismo, ya que el aumento de LH estimula mayor secreción de $\mathrm{E}_{2}$ por la célula de Leydigit ${ }^{14,32}$; en el hipogonadotropo se genera un desbalance E-T por disminución de $\mathrm{T}$, en presencia de niveles normales de $\mathrm{E}_{2}$ provenientes de la aromatización periférica de andrógenos suprarrenales ${ }^{3,7}$.

El alcoholismo influye en el nivel hipotalámico y testicular disminuyendo la secreción de testosterona e incrementa la transformación de andrógenos a estrógenos ${ }^{9}$.

La Gi de la realimentación y de la diabetes, se deberían al hipogonadismo hipogonadotropo funcional provocado por el compromiso nutricional y general; además influiría la «segunda pubertad» que ocurre con su recuperación 2,3,26,33.

En el caso sugerente de resistencia periférica a andrógenos, la unión de DHT al receptor fue normal, pero no se puede descartar alteración postreceptor o de su gen ${ }^{2,3}$. 


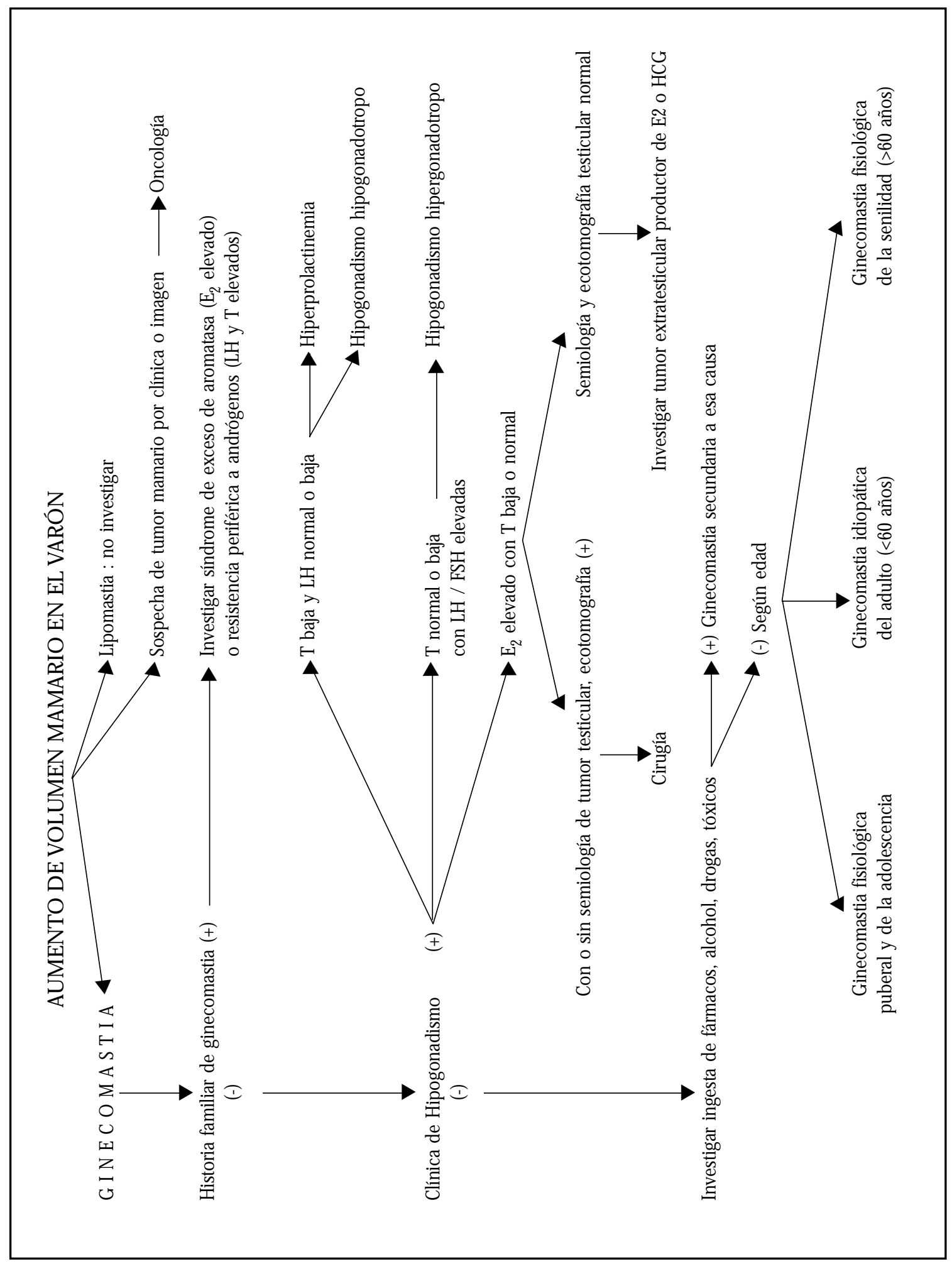


Los fármacos fueron causa importante de $\mathrm{Gi}$, especialmente los antiandrógenos que actúan por bloqueo del receptor de andrógeno; el acetato de ciproterona es, además, gestágeno, antigonadotropo y se administra con etinil estradiol ${ }^{34}$.

La cimetidina es también un antiandrógeno ${ }^{2}$; los digitálicos actuarían en el receptor de estrógenos; algunos anabólicos se pueden transformar en estrógenos y el ketoconazol es inhibidor de enzimas que intervienen en la síntesis de $\mathrm{T}^{2,3,14}$.

Sustancias químicas (xenoestrógenos) derivadas de plásticos, pesticidas e insecticidas ${ }^{35}$ contaminan el ambiente, se ligan al receptor de estrógenos o son disrruptores del eje HHT.

No pudimos comparar las causas de Gi encontradas en nuestro trabajo, pues las escasas series publicadas $^{9,11,17,30,31}$ se basan en poblaciones diferentes, sólo adultos o adolescentes, y no se utiliza el mismo criterio de clasificación.

Gran parte de las Gi encontradas fueron fisiológicas de la pubertad y adolescencia, diagnóstico de exclusión, ya que $21 \%$ de las Gi en

\section{REFERENCIAS}

1. Mathur R, Braunstein G. Gynecomastia: pathomechanism and treatment strategies. Horm Res 1997; 48: 95-102.

2. Wilson J, Aiman J, MacDonaid P. The pathogenesis of Gynecomastia. ADV Intern Med 1980; 25: 1-32.

3. Braunstein GD. Gynecomastia. N Engl J Med 1993; 328: $490-5$.

4. Sasano H, Kimura M, Shizawa S, Kimura N, Nagura H. Aromatase and Steroid Receptors in Gynecomastia and Male Breast Carcinoma: An Immunohistochemical Study. J Clin Endocrinol Metab 1996; 8: 3063-7.

5. Nydick M, Bustos J, Daie J Jr. Rawson R. Gynecomastia in Adolescent Boys. JAMA 1961; 178: 449-54.

6. Biro F, Lucky A, Huster G, Morrison J. Hormonal studies and physical maturation in adolescent gynecomastia. J Pediatr 1990; 116: 450-5.

7. MAHONey CP. Adolescent Gynecomastia. Pediatr Clin N Am 1990; 37: 1389-404.

8. NutTaL FQ. Gynecomastia as a Physical Finding in Normal Men. J Clin Endocrinol Metab 1979; 48: 338-40. menores de 20 años fueron patológicas; lo mismo ocurre en el adulto mayor con $80 \%$ de $\mathrm{Gi}$ patológicas.

$\mathrm{Si}$ consideramos que en esas edades la mayoría de las Gi son fisiológicas y no se estudian, se corre el riesgo de subdiagnosticar patologías que ameritan tratamiento específico (tumores) o terapia de reemplazo permanente como en los hipogonadismos hipo e hipergonadotropos.

El 39\% de las Gi patológicas no presentaban los síntomas y signos que obligarían a estudio, esto y lo anterior apoyan el planteamiento adoptado de investigar etiológicamente toda $\mathrm{Gi}$, con clínica acuciosa, solicitando estudio de laboratorio e imágenes en base a la semiología y valores de $\mathrm{E}_{2}$ y T.

Debe considerarse con cautela que ciertos grupos etarios sean estudiados someramente, por predominar en ellos las Gi fisiológicas o, que sólo ciertos síntomas y signos guíen el estudio, ya que arriesgamos subdiagnosticar un porcentaje importante de Gi patológicas.
9. CarLSON HE. Gynecomastia. N Engl J Med 1980; 303: 795-9.

10. Niewoehner C, NutTal F. Gynecomastia in a Hospitalized Male Population. Am J Med 1984; 77: 633-8.

11. Bowers S, Pearlman N, Mcintyre R, Finlayson C, HUERD S. Cost-effective Management of Gynecomastia. Am J Surg 1998; 176: 638-41.

12. WiLson JD. Enfermedades Endocrinas de la Mama. En: Harrison. Principios de Medicina Interna 14aㅡ Edición. Editores: Fauci, BraunwaldIsselbacher, Wilson, Martin, Kasper, Houser, Longo. McGraw-Hill. Interamericana de España, Madrid. 1998; 338: 2405-9.

13. Hands L, Greenall M. Gynaecomastia. Br J Surg 1991; 78: 907-11.

14. Grass AR. Gynecomastia. Endocrinol Metab Clin N Am 1994; 23: 825-37.

15. MacMilan D, Dixon M. Gynaecomastia: when is action required. The Practitioner 2000; 244: 785-7.

16. Vivanco X, Cuelo $X$, Abodovsky N, Sapunar J. Ginecomastia puberal: estudio clínico de 31 casos. Bol Hosp SJ de Dios 1990; 37: 9-12.

17. PABST I. Ginecomastia. Bol Hosp S J de Dios 1993; 40: 30-40. 
18. Mineur P, De Cooman S, Hustin J, Verhoeven G, De HeRTOGH R. Feminizing Testicular Leydig Cell Tumor: Hormonal Profile before and after Unilateral Orchidectomy. J Clin Endocrinol Metab 1987; 64: 686-91.

19. Kuhn J, Mahoudeau J, Biшaud L, Joly J, Rieu M, Gancel A et al. Evaluation of Diagnostic Criteria for Leydig Cell Tumours in Adult Men Revealed by Gynaecomastia. Clin Endocrinol 1987; 26: 407-16.

20. Braunstein GD. Aromatase and Gynecomastia. Endocrine-Related Cancer 1999; 6: 315-24.

21. No Authors Listed. Case records of the Massachusetts General Hospital. Weekly clinicopathological exercises. Case 12-2000. A 60 - year old Man with Persistent Gynecomastia after Excision of a Pituitary Adenoma. N Engl J Med 2000; 342: 1196204.

22. Einav-Bachar R, Phiшip M, Aurbach-Kupper Y, Lazar L. Prepubertal gynaecomastia: aetiology, course and outcome. Clin Endocrinol 2004; 61: 55-60.

23. Devoto E, Madariaga M, Lioi X. Factores Causales de Infertilidad Masculina. Contribución del Factor Endocrino. Rev Méd Chile 2000; 128: 184-92.

24. Danila D, Kubanski A. Prolactin Secreting Pituitary Tumors in Men. The Endocrinologist 2001; 11: 105-11.

25. Colao A, Loche S, Cappa M, Di Sarno A, Landi ML, Sarnacchiaro F et al. Prolactinomas in Children and Adolescents. Clinical Presentation and LongTerm Follow-up. J Clin Endocrinol Metab 1998; 83: 2777-80.
26. Hershrovitz E, Leiberman E. Gynecomastia: a review. The Endocrinologist 2002; 12: 321-32.

27. Pearson JC. Endocrinology of testicular Neoplasms. Urology 1981; 17: 119-25.

28. Young S, Gooneratne S, Straus F, Zeluer W, Serdar B, Rosenthal I. Feminizing Sertoli Cell Tumor in Boys with Peutz-Jeghers syndrome. Am J Surg Pathol 1995; 19: 50-8.

29. Connay G, MacConnell T, Wells G, Slater S. Importance of scrotal ultrasonography in Gynaecomastia. Br Med J 1988; 297: 1176-7.

30. Berkovitz G, Guerami A, Brown T, Macdonald P, Migeon C. Familial Gynecomastia with Increased Extraglandular Aromatization of Plasma Carbon19 Steroids. J Clin Invest 1985; 75: 1763-9.

31. HAMER DB. Gynaecomastia. Br J Surg 1975; 62: 326-9.

32. LPSETT MB. Physiology and Pathology of the Leydig Cell. N Engl J Med 1980; 303: 682-8.

33. Woolf P, Hamil R, McDonald J, Lee L, Keluy M. Transient Hypogonadotropic Hypogonadism Caused by Critical Illness. J Clin Endocrinol Metab 1985; 60: 444-50.

34. Devoto E, Aravena L. Utilidad terapéutica de los Antiandrógenos. Rev Méd Chile 1993; 12: 1183-90.

35. SkakKebaek N, LefFers H, Rajpert-De Meyts E, CARLSEN E, Grigor K. Should we watch what we eat and drink? report on the International workshop on Hormones and Endocrine disrupters in food and water: Possible impact on Human Health. Copenhagen, Denmark 27-30 May 2000; Trends Endocrinal Metab 2000; 11: 291-3.

Agradecimientos

Agradecemos a la doctora Soledad Hidalgo por la referencia de pacientes que incluyeron un tumor testicular. 